\title{
Making it rain: cloud-based molecular simulations for everyone
}

\author{
Pablo R. Arantes, ${ }^{*, \dagger, \perp}$ Marcelo D. Polêto, ${ }^{*, \dagger}$ Conrado Pedebos, ${ }^{*, \boldsymbol{\Phi}, \S}$ and Rodrigo \\ Ligabue-Braun*,\| \\ $\dagger$ Department of Bioengineering, University of California, Riverside, CA 92521, United \\ States \\ $\ddagger$ Department of Biochemistry, Virginia Tech, Blacksburg, VA 24061, United States \\ ISchool of Chemistry, University of Southampton, Highfield Campus, Southampton SO17 \\ 1BJ, United Kingdom \\ $\S$ Present Address: Department of Biochemistry, University of Oxford, Oxford OX1 3QU, \\ United Kingdom \\ || Department of Pharmacosciences, Federal University of Health Sciences of Proto ALegre \\ (UFCSPA), Porto Alegre 90050-170, RS, Brazil. \\ $\perp$ Corresponding author
}

E-mail: pabloa@ucr.edu; mdpoleto@vt.edu; conrado.pedebos@bioch.ox.ac.uk;

rodrigolb@ufcspa.edu.br 


\section{Supporting Information}

\section{List of Figures}

$1 \quad$ Structural analyses of MD simulations using PyTraj embedded in the note-

books: RMSD values over time (A), its distribution (B) and a 2D RMSD plot

(C); radius of gyration over time (D) and its distribution (E); Cross-correlation analysis (F); RMSF per residue (G); Principal Component Analysis (H) and its eigenvector distribution (I). . . . . . . . . . . . . . . . . . . . . . 3 

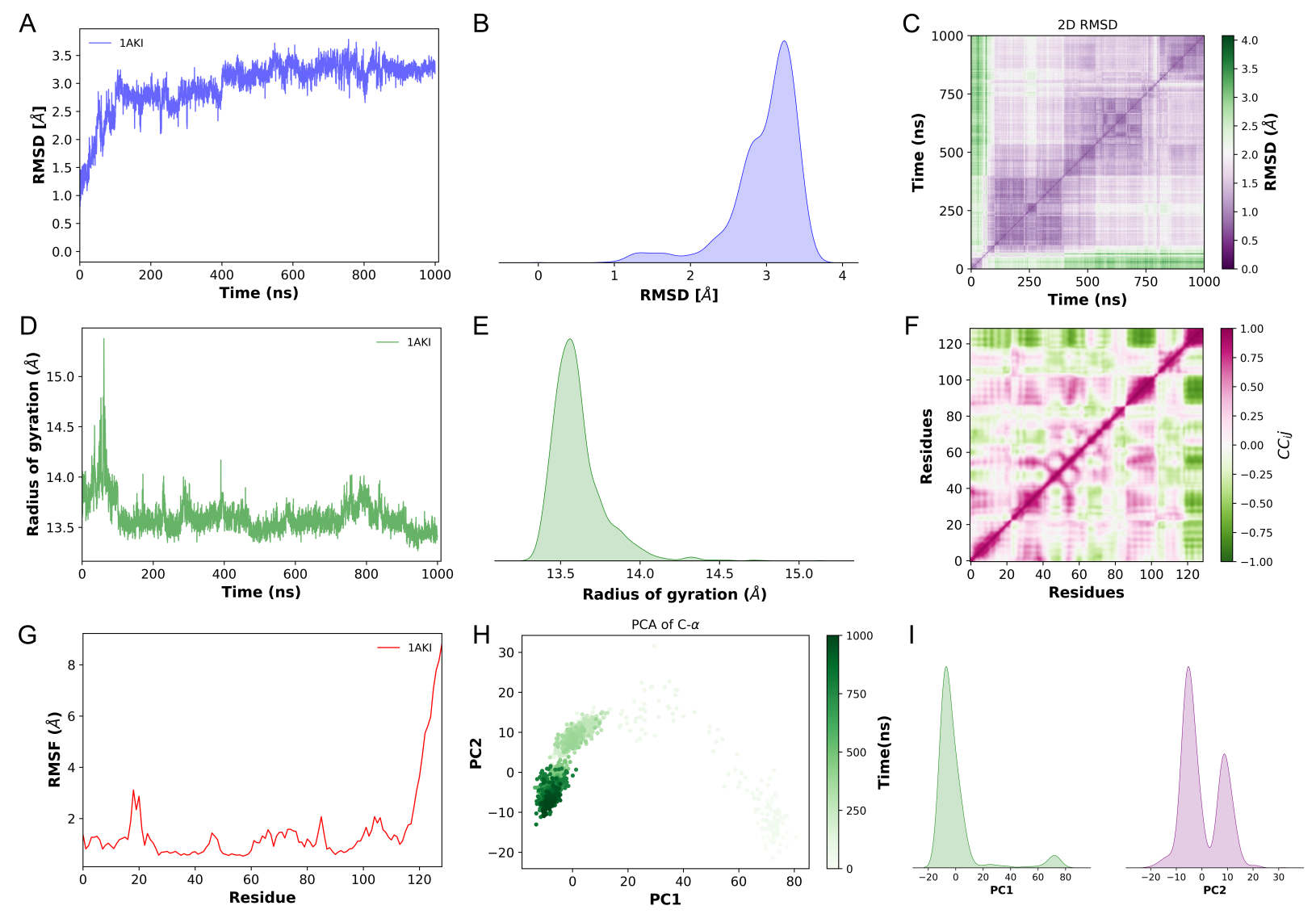

Figure S 1: Structural analyses of MD simulations using PyTraj embedded in the notebooks: RMSD values over time (A), its distribution (B) and a 2D RMSD plot (C); radius of gyration over time (D) and its distribution (E); Cross-correlation analysis (F); RMSF per residue (G); Principal Component Analysis (H) and its eigenvector distribution (I). 